\title{
Improving Vigor in shrunken-2 Corn Seedlings
}

\author{
Carlos A. Parera, Daniel J. Cantliffe, D.R. McCarty, and L. Curtis Hannah \\ University of Florida, Institute of Food and Agricultural Sciences, Horticultural Sciences Department, \\ P.O. Box 110690, Gainesville, FL 32611-0690
}

Additional index words. germination, maize, Zea mays, $B$ chromosome, conductivity, leakage

\begin{abstract}
The shrunken-2 (sh2) mutant of maize (Zea mays L.) increases sucrose and reduces starch in developing endosperm. An associated trait is poor seed and seedling vigor in seeds containing the mutation. The specific effects of $s h 2$ mutant endosperm on embryo and seedling vigor were determined by analyzing seeds that contained either concordant wild-type or nonconcordant combinations of mutant and wild-type embryo and endosperm genotypes. The nonconcordant seed types that contained a wild-type embryo in association with a $\operatorname{sh} 2$ mutant endosperm or a sh 2 mutant embryo in association with a wild-type endosperm were generated using the TB-3La translocation chromosome in which a wild-type $S h 2$ gene is attached to the centromeric portion of a $B$ chromosome. Under stress conditions (complex stress vigor test), the seeds with mutant endosperm had lower germination, seedling fresh and dry weight, and index of conductivity than seeds with wild-type endosperm. Mutant endosperm and embryos excised from mutant endosperm imbibed more water than wild-type endosperm or embryos excised from wild-type endosperm. Because of the high concentration of osmotic solutes in the mutant endosperm, a rapid water uptake may induce a membrane disorganization. Leachate conductivities of seeds with mutant endosperm were higher than seeds with wild-type endosperm. In addition, a higher sucrose content and a lower raffinose to sucrose ratio were measured in the wild-type embryos associated with mutant endosperms than in the normal embryos excised from concordant wild-type seeds. These results suggest that a high rate of water uptake caused by the elevated concentration of osmotic solutes in seeds with mutant endosperms may affect membrane integrity during imbibition. Alternatively, the lower raffinose to sucrose ratio present in the mutant endosperm class might affect stabilization of cell membranes during seed desiccation. Embryos cultured in media containing $10 \%$ starch or no carbohydrate produced smaller seedlings than embryos cultured in $5 \%$ or $10 \%$ sucrose. Wild-type embryos excised from mutant endosperms exhibited lower germination in $0 \%$ and $5 \%$ sucrose media than embryos from concordant seed, indicating that reduced water uptake rates associated with lower external osmotic potential (10\% sucrose) can improve vigor of embryos associated with sh2 mutant endosperm. The reduced vigor of embryos and seedlings that develop in association with sh 2 mutant endosperm can be traced to the physiological and biochemical effects of the elevated sucrose levels present during seed formation and imbibition.
\end{abstract}

The mutant shrunken-2 (sh2) gene modifies the synthesis of carbohydrates in maize (Zea mays) endosperm, increasing the levels of sucrose and decreasing starch at maturity (Creech, 1956). Tsai and Nelson (1966) reported that the Sh2 gene, located in the long arm of chromosome 3 , affects the activity of adenosine diphosphoglucose pyrophosphorylase (ADP-glucose pyrophosphorylase) in the endosperm. The $S h 2$ gene has been cloned and encodes one subunit of ADP-glucose pyrophosphorylase (Bhave et al., 1990). This enzyme plays a key role in starch biosynthesis, catalyzing the reaction ATP + glucose-1-phosphate to form ADP-glucose + PPi (Preiss, 1978). Kernels carrying the sh2 mutation have less starch and 7 to 10 times more sucrose at maturity than normal genotypes (Creech, 1956). The sh2 mutant does not modify the enzyme levels in the embryo (Hannah and Nelson, 1976).

Seeds of sh2 maize are less uniform, lighter, and easier to damage than wild type seeds (Styer and Cantliffe, 1983). Poor seed and seedling vigor is a characteristic of cultivars carrying the $s h 2$ gene. Wann (1980) concluded that the lower seed vigor was related to small endosperm. Styer and Cantliffe (1984a) reported a dysfunction of the scutellum in relation to carbohydrate metabolism and use. Harris and DeMason (1989) concluded that the low vigor is associated with inadequate aleurone-controlled reserve mobilization. Parera and Cantliffe (1991) improved germination of sh2 sweet corn hybrids by controlling water uptake. Although alterations in the scutellum, seed weight, and catabolism of seed

Received for publication 15 Mar. 1996. Accepted for publication 17 July 1996. Florida Agricultural Experiment Station journal series R-04623. The cost of publishing this paper was defrayed in part by the payment of page charges. Under postal regulations, this paper therefore must be hereby marked advertisement solely to indicate this fact. reserves are noted changes in $s h 2$ seeds, the direct effect of the mutation on germination and subsequent seedling vigor is not clearly understood.

Increased seed leakage after imbibition has been associated with membrane damage in cotyledons of soybean (Schoettle and Leopold, 1984). Heat-killed embryos have greater water uptake due to changes in fluidity or ordering of the membranes (Murphy and Noland, 1982). Koster and Leopold (1988) proposed that raffinose may prevent crystallization of the sucrose during dehydration, improving membrane protection and stabilization. Seed vigor of maize embryos was correlated with raffinose concentration (Bernal-Lugo and Leopold, 1992). A high raffinose to sucrose ratio in maize seeds was highly correlated with membrane stability during drying (Chen and Burris, 1990).

The characteristics of the endosperm affect the embryo during germination and subsequent seedling growth. The structure and chemical composition of the endosperm may alter the quality and quantity of the metabolites available for the embryo, seed osmotic potential, and germination. The embryo is responsible for the initial growth of the axes and subsequent control of reserve mobilization (Bewley and Black, 1978). Poor germination and seedling vigor of $s h 2$ seeds is an enigma. All current molecular and biochemical data show that the $s h 2$ gene is expressed only in the endosperm. Although the embryo and seedling contain starch and have ADP-glucose pyrophosphorylase activity, this activity is independent of the $s h 2$ mutation in seedlings.

Clearly, seedling vigor is reduced as a consequence of the seed mutation. This may be explained by the association between the endosperm and embryo as discussed above. The question can be addressed by creating seeds that contain a mutant endosperm and wild-type embryo. If the poor germination of $s h 2$ is due to endosperm, then the genetic constitution of the $s h 2$ locus in the 
embryo should have no affect on subsequent reduction in seed and seedling vigor. To test this hypothesis, a mechanism to create such seeds was used.

Supernumerary or $B$ chromosomes are nonessential to the maize plant and are only found in certain maize lines. This chromosome disjoins normally in virtually all mitotic events. However, it has been noted (Roman and Ullstrup, 1951) that $B$ chromosomes commonly undergo a nondisjunction at mitosis giving rise to two sperm. As a consequence, the two sperm are genetically dissimilar: one sperm contains two copies of the $B$ chromosome while the other contains none. It has also been shown that translocations involving the portion of the chromosome containing the $B$ centromere, plus a chromosome segment for any member of the $A$ complement of chromosomes, show this pattern of nondisjunction. TB-A translocations involving the long arm of chromosome 3 that contain the Sh2 locus are available; thus, it is possible to create seeds with wild-type embryo and mutant endosperm. Such seeds arise when the sperm containing the two $B$ chromosomes fertilizes the egg to give rise to the embryo, while the sperm containing no $B$ chromosomes fuses with the polar nuclei to give rise to an endosperm lacking a functional $S h 2$ gene.

Because sh2 has no visible effect on the embryo, it was necessary to identify a gene extremely closely linked to $S h 2$, whose expression could be followed in the embryo. The $A l$ locus fulfills this criterion. The $A l$ locus is involved in anthocyanin biosynthesis and can be scored in the embryo by the presence or absence of pigment. Furthermore, the $A$ and $S h 2$ locus are very closely linked $(0.2 \%$ recombination $)$. Therefore, seeds containing the proper complement of other genes involved in anthocyanin biosynthesis and the $A$ locus in the embryo but not in the endosperm will show pigmentation in the embryo and will be colorless in the endosperm. Furthermore, fertilization of the opposite type involving the sperm arising from the nondisjunction event will give rise to a pigmented endosperm but colorless embryo. Incorporation of the $R-n j$ allele (see material and methods), which pigments only the crown of the endosperm, allows one to easily distinguish the presence or absence of pigment in both tissues of the seed.

Since the recombination between $a l$ and $s h 2$ is rare $(0.2$ map unit), we can, therefore, confidently classify the genotype of the embryo and the endosperm in nonconcordant kernels by pigmentation. The sh2 genotype of the endosperm can be classified on the basis of plump or shrunken seed. We exploited such seed to examine germination under stress and nonstress conditions and also some of the physiological parameters that have, in past studies, been associated with altered seed and seedling vigor. Our objectives were to 1) determine whether the genetic constitution at the $s h 2$ locus in the embryo has any effect on seed and seedling vigor and 2) evaluate the effect of the $\operatorname{sh} 2$ mutant endosperm on the embryo in some of the early physiological steps of germination and seedling development to clarify their relationship to poor seed vigor.

\section{Materials and Methods}

Seeds used in this experiment were derived ultimately from a cross of yellow (lacking anthocyanin) female parent to a pigmented stock containing the TB-A translocation. The pedigrees of the parents are the following. The female parent was derived from a cross of 'Florida Stay Sweet' (genetic constitution of chromosome 3 is al sh2/al sh2) to a pigmented stock generously supplied by Ed Coe, Univ. of Missouri. The genetic constitution of chromosome 3 of this latter stock is Al Sh2/al Sh2. The stock also contained the alleles $R-n j$ and $r$ on chromosome 10. The $R$ locus, like the $A$ locus, is involved in anthocyanin biosynthesis. $R-n j$, when combined with appropriate other genes, pigments the crown of the kernel and embryo but does not pigment the aleurone layer overlying the embryo. As a result, classification of embryo and endosperm pigmentation is unambiguous. Seeds homozygous for the $r$ allele lack anthocyanin. Colorless seed from the above cross were chosen for the subsequent cross to the TB-A translocation stock. These seed were chosen to enhance the frequency of alSh2/ alsh 2 seeds. However, colorless seed containing the $A 1$ allele could also arise via homozygosity for a recessive allele at one of the other four loci involved in anthocyanin biosynthesis. Because heterozygosity commonly exists for these five genes involved in anthocyanin biosynthesis among genetically useful maize lines, and because the genotype at all of these loci is not known for certainty for these two stocks, the colorless seed resulting from the above cross potentially could be of various genotypes. The final classification scheme, as detailed below, considered this.

The TB-A translocation stock was initially obtained from the Maize Genetics Cooperation Stock Center. To incorporate appropriate anthocyanin markers, the original TB-A stock was initially crossed as female with a stock containing $R-n j$. The rationale for incorporation of $R-n j$ was given above.

Following the cross of the $\operatorname{sh} 2$ containing stock by the TB-A containing stock, seed of the following phenotypes were chosen for use in these experiments.

Class 1: Seeds containing a colorless shrunken endosperm and a colored embryo. These arose from the fusion of the al sh 2 eggs and polar nuclei with nonconcordant sperm. Here, the TB-Acontaining sperm fused with the egg while the TB-A-lacking sperm fused with the polar nuclei.

One can infer, with very high probability, that the embryos contain the functional or wild-type allele of the $S h 2$ locus since $A l$ and $S h 2$ are only 0.2 map units apart. Recombination between these genes can be observed since this would give rise to pigmented shrunken endosperms. None was observed in any of the crosses involved in these experiments.

Class 2: Seeds containing colorless plump endosperms and a colored embryo. These arose from the fusion of al Sh2 eggs and polar nuclei with the nonconcordant sperm as described for Class 1 seed above. These seeds are genetically identical to those of Class 1 except for the substitution of $S h 2$ for $s h 2$. Hence, these serve as the proper control for monitoring the effect of $s h 2$ mutation in the endosperm on embryo development and subsequent seed and seedling vigor.

Class 3: Seeds containing a colored plump endosperm and a colorless embryo. These arose from fertilization events of the opposite type of those described from Classes 1 and 2 seed above. Here, the TB-A containing sperm fused with the polar nuclei, while the corresponding sperm fused with the egg. The $a 1$ allele is contributed by the female, although the genetic contribution of the female parent at the sh2 locus cannot be ascertained. On average, the $\operatorname{sh} 2$ and $S h 2$ alleles should be equally distributed within this class.

Other noninformative classes of seed were also obtained and were discarded. These resulted from normal disjunction of the TBA chromosome, transmission of the $A l$ allele from the female parent, or homozygosity for recessive alleles at one of the other pigment genes.

Seeds were produced under greenhouse conditions to reduce pathogen infection. Ears were dried under controlled conditions $\left[30{ }^{\circ} \mathrm{C}\right.$ and $25 \%$ relative humidity $\left.(\mathrm{RH})\right]$ to $9 \%$ to $10 \%$ moisture content. After drying, the seeds were classified according to the phenotypes described above. Seeds were stored at $15^{\circ} \mathrm{C}$ and $45 \%$ 
Table 1. Germination percentage, seedling fresh weight (FW) and dry weight (DW) after complex stress vigor test, and index of conductivity and stress vigor test (ICS) of concordant and nonconcordant corn seeds.

\begin{tabular}{lccccrr}
\hline \hline Class & $\begin{array}{c}\text { Shrunken2 } \\
\text { endosperm }\end{array}$ & $\begin{array}{c}\text { Genotype } \\
\text { embryo }\end{array}$ & $\begin{array}{c}\text { Germination } \\
(\%)\end{array}$ & $\begin{array}{c}\mathrm{FW}^{\mathrm{z}} \\
(\mathrm{g})\end{array}$ & $\begin{array}{c}\mathrm{DW}^{\mathrm{z}} \\
(\mathrm{g})\end{array}$ & \multicolumn{1}{c}{$\mathrm{ICS}^{2}$} \\
\hline 1 & Mutant & Normal & 59 & 0.191 & 0.0143 & 2.84 \\
2 & Wild type & Mutant/normal & 97 & 0.414 & 0.0299 & 21.38 \\
3 & Wild type & Normal & 100 & 0.515 & 0.0277 & 22.07 \\
LSD $_{(0.05)}$ & & & 13 & 0.105 & 0.0082 & 0.99 \\
\hline
\end{tabular}

${ }^{\mathrm{z}}$ Average morphologically normal plants.

yWild type or normal.

RH before the experiments. In experiments that require separated embryos, the axes and scutellum were hand dissected from the dry seeds.

Complex stress vigor test. Twenty-five seeds were soaked in 35 $\mathrm{mL}$ of water at $25^{\circ} \mathrm{C}$ for $24 \mathrm{~h}$ then moved to $5{ }^{\circ} \mathrm{C}$ for another 24 h. Seeds were sown on a three-layer germination paper (Anchor Paper Co., St. Paul, Minn.) moistened with distilled water and incubated at $15^{\circ} \mathrm{C}$ for $4 \mathrm{~d}$ in a closed plastic container $(21.5 \times 32.5$ $\times 5.5 \mathrm{~cm}$ ) following the procedures of Barla-Szabo and Dolinka (1988). The electrical conductivity of the leachate was recorded after each $24 \mathrm{~h}$ soak. Germination percentage was recorded after the second soak and fresh and dry weights were taken. The index of conductivity and stress vigor test (ICS) was calculated after each stress test.

Leachate conductivity and seed imbibition. Twenty-five seeds were soaked in $20 \mathrm{~mL}$ of distilled water at $25{ }^{\circ} \mathrm{C}$ for $9 \mathrm{~h}$. The electrical conductivity of the leachate was measured every hour with a conductimeter (Lecto MhoMeter; Lab Line Instruments, Melrose Park, Ill.) and expressed as $\mu \mathrm{mhos} \cdot \mathrm{g}^{-1}$ of seed. The fresh weight was determined 3,6 , and $9 \mathrm{~h}$ after soaking. The embryo and endosperm were excised, weighed, and oven dried $\left(75^{\circ} \mathrm{C}\right)$ for $72 \mathrm{~h}$.

Pericarp thickness and embryo to endosperm ratio. Seeds were imbibed $18 \mathrm{~h}$ in a (v/v) solution of 1 glycerol : 2 water. The crown cap and tip cap were cut, and the pericarps were gently excised from the kernel and dried at room temperature $\left(25 \pm 1^{\circ} \mathrm{C}\right)$ for $4 \mathrm{~h}$ on a Whatman no. 3 filter paper (Martin et al., 1980). The thickness was evaluated with a micrometer at the center and the two extremes of the pericarp strip and expressed as the average of the three measures. The embryo to endosperm ratio was calculated for each type of seeds on a fresh weight basis.

Embryo culture. Excised embryos were surface sterilized $3 \mathrm{~min}$ in a $1.5 \%(\mathrm{v} / \mathrm{v})$ sodium hypochlorite solution and rinsed three times with sterile water. Four embryos were sown in a plastic box $(6.5 \times$ $6.5 \times 9.8 \mathrm{~cm}$ ) on a Whatman no. 3 filter paper. The boxes were filled with a $20 \mathrm{~mL}$ of Murashige and Skoog (1962) medium, adjusted to $\mathrm{pH} 6.5$, containing $10 \%$ sucrose $(\mathrm{w} / \mathrm{v}), 5 \%$ sucrose, $10 \%$ starch, or no carbohydrates. The embryos were placed at $25^{\circ} \mathrm{C}$ in the dark. After $4 \mathrm{~d}$, germination percentage was calculated, and fresh and dry weight of the seedlings was measured.

Sugar characterization. Excised endosperms and embryos were separately homogenized (Virtis homogenizer; Virtis Co., Gardiner,
N.Y.) at high speed with $10 \mathrm{~mL}$ of $80 \%(\mathrm{v} / \mathrm{v})$ ethanol. The homogenate was centrifugated at $20,000 \times g$ for $10 \mathrm{~min}$ and the supernatant was separated. The pellet was washed with $5 \mathrm{~mL}$ of $80 \%$ ethanol and recentrifugated (Chen and Burris, 1990). Both supernatants were combined and the ethanol was evaporated in vacuo at $30^{\circ} \mathrm{C}$. The volume was adjusted to $5 \mathrm{~mL}$ with water and filtered through a $0.2-\mu \mathrm{m}$ nylon filter. The sugars were characterized by HPLC (BioRad Chemicals, Richmond, Calif.), where the peaks of interest were compared with standard peaks. An Aminex carbohydrate HPX-87C column was used with water as mobile phase at a flow rate of $0.6 \mathrm{~mL} \cdot \mathrm{min}^{-1}$. The sugar concentrations were expressed as $\mathrm{mg} \cdot \mathrm{g}^{-1}$ of fresh weight.

\section{Results}

The genetic materials analyzed here were created to monitor the consequence of loss of sh2 function in the embryo or in the endosperm on subsequent seed and seedling vigor. After the complex stress test, the final germination percentage of seeds with mutant endosperms/normal embryos (Class 1) was almost $40 \%$ lower than that of the seeds with nonshrunken endosperms of either Class 2 or 3 (Table 1). Furthermore, the depressing effect of the mutant endosperm was seen in the resultant seedling fresh and dry weights following $4 \mathrm{~d}$ of germination. Thus, the detrimental effect of the mutant endosperm is seen even in seeds capable of germination and normal growth. The index of conductivity and stress test, which is a good predictor of field emergence (Parera, et al. 1995), was significantly lower in seeds with mutant endosperm.

Embryo weight was not affected by the endosperm genotype (data not shown). However, seeds with mutant endosperm were smaller and lighter, and the endosperm to embryo ratio was almost three times higher in wild-type endosperm seeds (Table 2). All seeds used arose from the same maternal parent, and, since the pericarp is a maternally derived tissue, we do not expect differences in the pericarp thickness. As anticipated, significant differences in pericarp thickness were not observed. The kernels were separated manually from the cob and dried at low temperature to avoid cracks or fissures in the pericarp and, thus, minimize potential modification of imbibition and leachate conductivity.

The electrolyte leakage in seeds with mutant endosperm was significantly higher than the wild-type counterparts and was not

Table 2. Pericarp thickness (PT) and endosperm to embryo ratio of concordant and nonconcordant corn seeds.

\begin{tabular}{lcccc}
\hline \hline & Shrunken-2 & Genotype & PT & \multicolumn{2}{c}{ Endosperm : embryo } \\
Class & endosperm & embryo & $\mu \mathrm{M}$ & 2.82 \\
\hline 1 & Mutant & Normal & 67.0 & 9.74 \\
2 & Wild type & Mutant/normal & 75.1 & 8.68 \\
3 & Colorless & Normal & 72.6 & 1.80 \\
LSD $_{(0.05)}$ & & & NS & \\
\hline
\end{tabular}

${ }^{\mathrm{z}}$ Wild type or normal. 


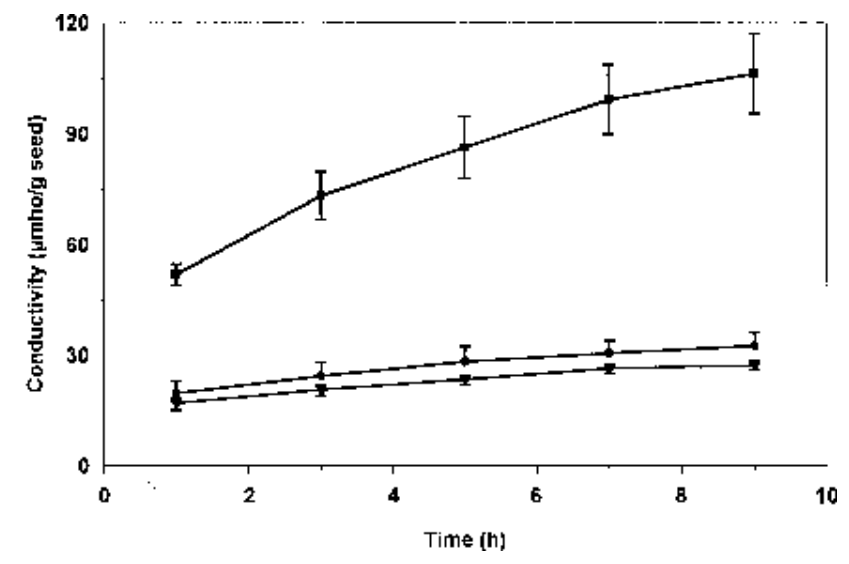

Fig. 1. Leakage conductivity of seeds. Mutant endosperm/normal embryo (ם), wild-type endosperm/mutant/normal embryo (-), and wild-type endosperm/ normal embryo $(\boldsymbol{\nabla})$. Data represent the mean of four measurements.

affected by the embryo genotype (Fig. 1). The water uptake by embryos excised from seeds with mutant endosperm was higher than embryos excised from wild-type seeds regardless of their genotype (Fig. 2a). Similarly, the mutant endosperm imbibed more water than wild-type seed (Fig. 2b).

When the embryos were germinated on different culture media, wild-type embryos excised from mutant endosperm had lower germination than the same genotype embryos excised from wildtype endosperm in no carbohydrate or $5 \%$ sucrose (Fig. 3a). However, embryos germinated in sucrose (especially 10\%) produced more-vigorous seedlings (higher dry weight and seedling length, data note shown) compared to starch (10\%) or noncarbohydrate media (Fig. 3b).

A sugar analysis was done to determine differences in endosperm and its effect on the embryo. Two types of seeds were used: mutant endosperm/wild-type (colored) embryo and wild-
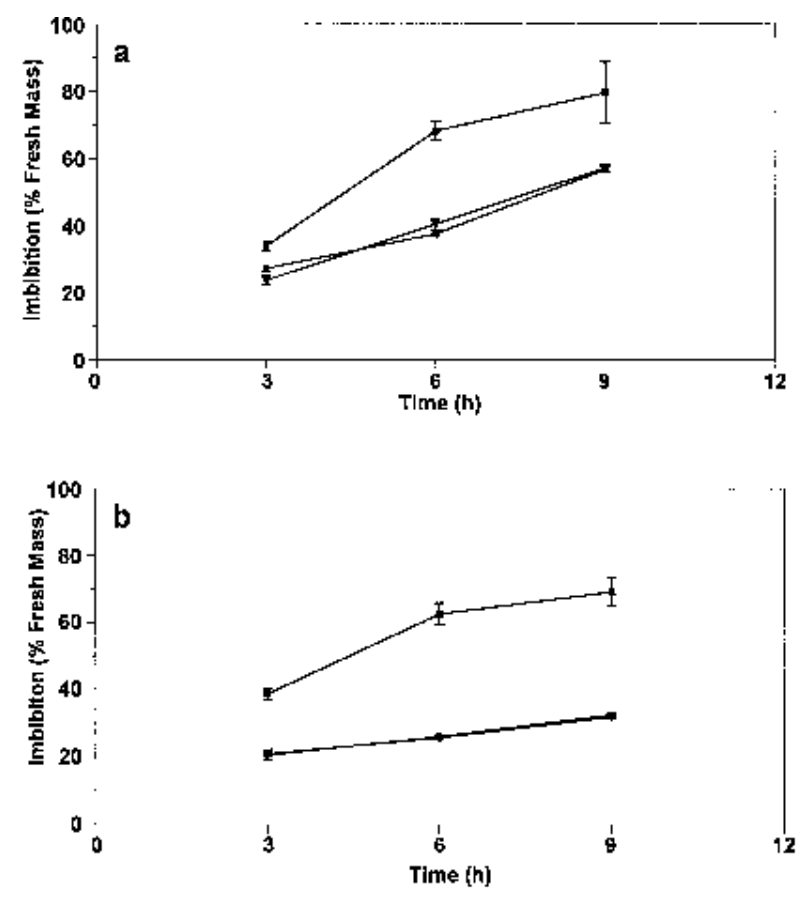

Fig. 2. Imbibition of embryo (a) and endosperm (b) of seeds. Mutant endosperm/ normal embryo ( $\mathbf{\square})$, wild-type endosperm/mutant-normal embryo $(\boldsymbol{\nabla})$, and wildtype endosperm/normal embryo $(\mathbf{)})$. Data represent the mean of four measurements. type (colorless) endosperm/wild-type (colored) embryo. Levels of sucrose, raffinose, and reducing sugars (glucose and fructose) in embryo and endosperm reflect the particular tissue under investigation. The amount of sucrose in embryos was almost four times higher than that in the endosperm (Fig. 4). Embryos from seeds with mutant endosperm had a higher sucrose concentration and reduced raffinose levels than embryos from wild-type endosperm seeds (Fig. 4a). No glucose was detected in the embryo, and no significant differences in fructose concentration were observed. The amount of sucrose and raffinose of mutant endosperms was higher than that of the wild type (Fig. 4b). Glucose and fructose were not detected in wild-type endosperms. The raffinose to sucrose ratio in the embryo was modified by the endosperm genotype (Fig. 5). Wild-type embryos excised from mutant endosperms had lower ratios than embryos excised from wild-type endosperm. Wild-type endosperm also had higher ratios than the mutant.

\section{Discussion}

These experiments were designed to determine if a reduction in seed vigor of sh2-containing maize seedlings was due to the genotype of the endosperm or embryo. The experiments were made possible by exploiting a translocated chromosome in which the $S h 2$ locus and the closely linked $A l$ locus were paternally transmitted to the embryo but not the endosperm. When the maternally transmitted chromosome contains recessive alleles of both loci, the resulting seed can be classified since the endosperm is colorless/shrunken whereas the embryo is colored by the presence of the $A l$ allele. In addition to this class, the incorporation of a maternally derived $S h 2$ allele gives rise to seed in which the endosperm and embryo are wild type at the Sh2 locus. Since all of the seeds are produced on the same set of ears and have common parents, differences in seedling vigor arising from heterogeneity at unlinked loci or environmental effects on parental plant growth should be randomized among the various populations of seeds.

Biochemical and molecular analyses showed that Sh2 is not expressed in the embryo, and the only tissue of $S h 2$ expression in maize seed is in the endosperm. Experiments reported from various laboratories (Dickinson and Preiss, 1969; Hannah and Nelson, 1976) confirm the fact that ADP-glucose pyrophosphorylase is not reduced in sh 2 mutant embryos. Only the endosperm specific form of this enzyme is affected by $\operatorname{sh} 2$ mutants. Molecular cloning and expression of the $\mathrm{SH} 2$ protein in $\mathrm{E}$. coli followed by antibody production have allowed an analysis of the gene at the molecular level (Giroux and Hannah, 1994; Giroux et al., 1994). The $S H 2$ protein is not detectable in the embryo, and a transcript with only a weak homology to $S h 2$ is found in the embryo. Molecular cloning and sequencing of this transcript (Giroux et al., 1995) shows that it is not identical to the Sh2 transcript found in the endosperm. The conclusion that $S h 2$ does not encode this transcript was confirmed by the lack of linkage between the locus (AGP1) encoding this transcript (Burr et al., 1991) and chromosome 3 . To conclude, all molecular and biochemical data clearly point to the lack of $s h 2$ expression in the embryo. On the basis of these analyses, it was expected that the reduced seedling vigor would be the result of the mutant endosperm and would be unaffected by the presence or absence of the functional $S h 2$ allele in the embryo. The data presented here are in clear accord with this expectation.

Seed germination is a complex mechanism involving several biochemical and physiological processes. The first step of seed germination is rehydration. Chemical composition of the seed, 


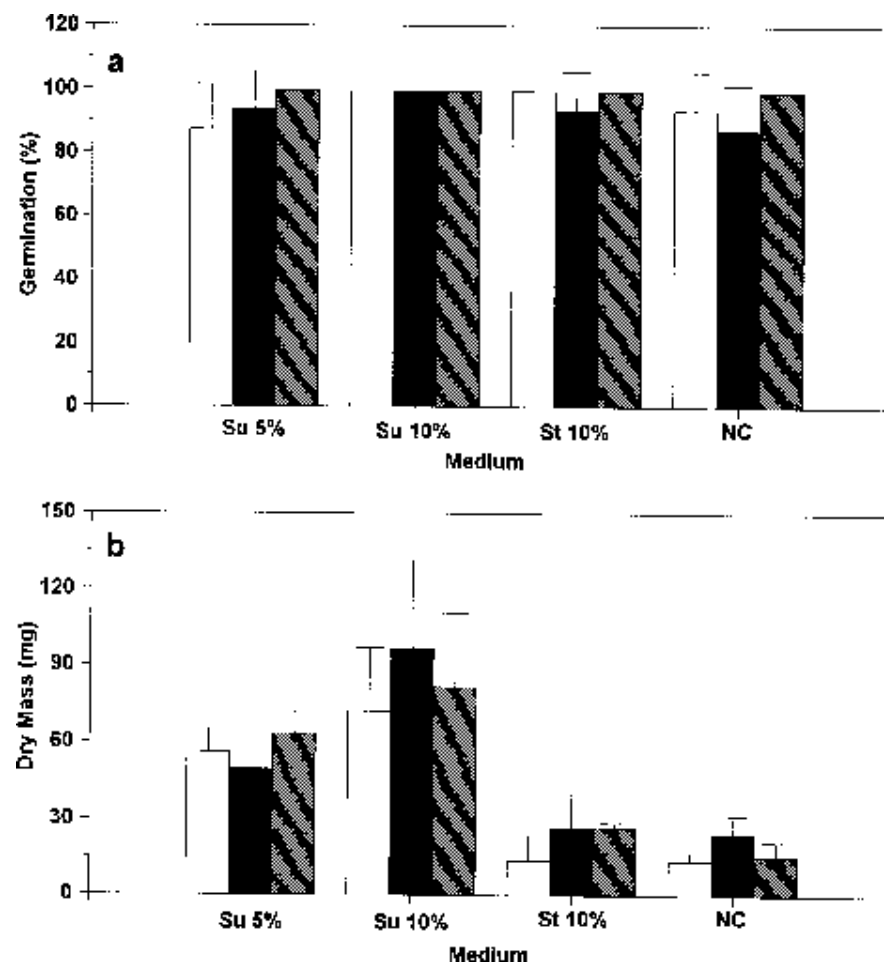

Fig. 3. Germination (a) and dry mass of the seedlings (b) in different media of embryos from nonconcordant seeds. Mutant endosperm/normal embryo (unfilled bar), wild-type endosperm/mutant-normal embryos (solid bar), and wild-type endosperm/normal embryo (hatched bar). Data represent the mean of four measurements. $\mathrm{Su}=$ sucrose, st-starch, $\mathrm{NC}=$ no added carbohydrates.

membrane permeability, seed size, presence and/or condition of the seed coat, and physical constants are all factors controlling seed water uptake (Woodstock, 1988). The pericarp is the outermost structure and is known to affect water movement in and out of the seed (Crane et al., 1959). In these experiments, difference in pericarp thickness was not observed; therefore, differences in water uptake and electrical conductivity are not likely to be a direct consequence of thickness. The higher imbibition rate in seeds with mutant endosperm was a probable consequence of their high osmotic solute concentration. As noted in the results, seeds with mutant endosperm had about four times more sucrose concentration in the endosperm than seeds with wild-type endosperm. In a previous report (Styer and Cantliffe, 1984a), the total sugar concentration in the whole seed and embryo of homozygous sh 2 seed was higher than that in normal genotype seeds.

As in previous reports (Tracy and Juvik, 1988; Wann, 1980) the electrical conductivity of the leachate was higher in seeds with mutant endosperm. The rate of electrolyte leakage in seeds is related to membrane leakiness and intactness (Woodstock, 1988). This structure is severely stressed during dehydration or hydration. Sucrose plays an important role in membrane protection (Crowe et al., 1984). Koster and Leopold (1988) reported that the ability of the seed axis to tolerate desiccation is correlated with the presence of sucrose combined with oligosacharides (raffinose and stachyose). The role of raffinose is critical in avoiding the crystallization of sucrose and it promotes onset of the glass stage, a condition for seed desiccation tolerance (Koster, 1991). Sucrose concentration was greater in mutant endosperm and wild-type embryos excised from mutant endosperms. However, the raffinose to sucrose ratio of the embryos was higher in wild-type endosperm and embryos excised from wild-type endosperms.
The medium with no carbohydrates had a higher (less negative) water potential than the other media. The higher water potential could induce a more rapid water uptake by embryos. The embryos derived from mutant endosperm took up water more rapidly and had lower raffinose to sucrose ratio. They may have less tolerance to rapid water uptake, and the low germination and seedling vigor could be a consequence of damage during imbibition. Styer and Cantliffe (1984a) also reported a lower germination of sh2 embryos excised from $s h 2$ endosperm when the medium had no carbohydrates compared to a medium with $10 \%$ sucrose. Recently, two reports (Chern and Sung, 1991; Parera and Cantliffe, 1991) concluded that controlled seed water uptake improved germination and seedling vigor in sh 2 maize hybrids. When the seeds were placed under stress conditions in the stressing vigor test, the detrimental effect on germination and leakage of $s h 2$ seeds was more prevalent.

In a sweet corn hybrid containing the sh2 mutant endosperm, Sanwo and DeMason (1992) found reduced amylase activity in the aleurone layer. This possibly delayed starch degradation during germination. They suggested that the endomembrane system (RER, polysomes, Golgi, and mitochondria) in aleurone layer cells affect amylase synthesis and secretion. Secretion and mobilization of cell reserves in aleurone cells of barley involve dynamic membrane events (Fernandez and Staehelin, 1985). Aged seed leaks cytoplasm components to the external medium because of loss of the integrity of plasma membrane (Parrish and Leopold, 1978). The dysfunction of the scutellum to reserve mobilization previously reported by Styer and Cantliffe (1984a) in sh2 seeds could be attributed also to alteration of membrane systems by a rapid water uptake. High electrolyte leakage and imbibition rate were observed in seeds with $s h 2$ endosperm. This may represent alteration of the membrane system, which then may affect amylase synthesis and secretion. As a secondary effect, the higher amount of electrolytes lost by the seed could promote growth of pathogens in and around the seeds. This is a common symptom reported in $s h 2$
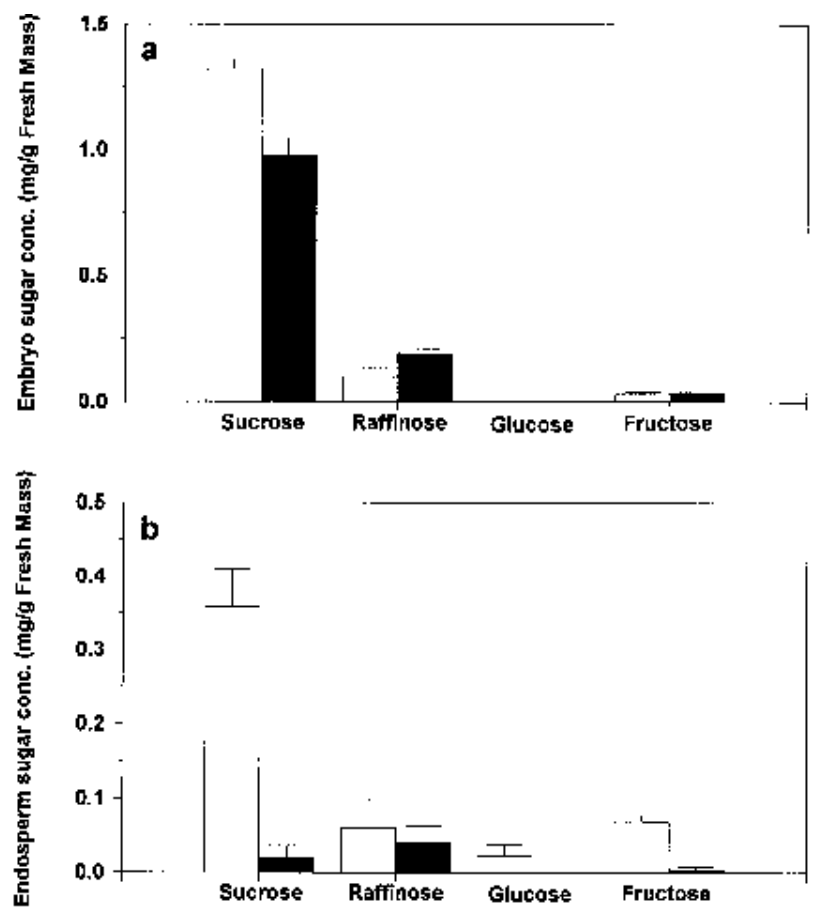

Fig. 4. Sugar concentration in embryos (a) and endosperms (b) of nonconcordant seeds. Mutant endosperm/mutant/normal embryo ( $\square$ ), wild-type endosperm/ normal embryos (ם). Data represent the mean of four measurements. 


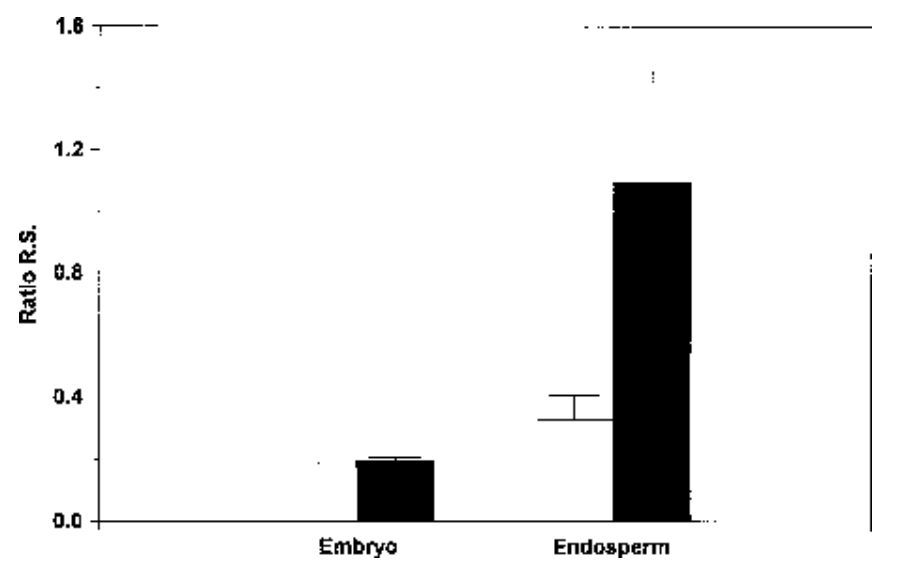

Fig. 5. Ratio raffinose/sucrose in embryos and endosperms of nonconcordant seeds. Mutant endosperm/normal embryo ( $\square$ ), wild-type endosperm/normal embryo a). Data represent the mean of four measurements.

hybrids (Berger and Wolf, 1974, Styer and Cantliffe, 1984b).

Several factors combined probably account for the poor seed and seedling vigor observed in seeds with sh2 endosperm. The membrane system plays a key role in the regulation of several biochemical processes in seed germination. Fragile membrane system (high electrolyte leakage and imbibition rate), no adequate membrane protection (sugar quality), and high osmotic potential (high levels of osmotic solute) in the endosperm could interact to alter the membrane system of the $s h 2$ endosperm seeds, leading to poor seed and seedling vigor. Consistent with the change in leakage (and the inferred change in membranes) is the possibility that alterations occur during seed development as a consequence of the high moisture content of the developing mutant seed. Because of the high sugar concentration, greater-than-wild-type levels of water are maintained during the development/maturation process of mutant seed. Perhaps, loss of the excess moisture during seed maturation causes membrane damage and reduced vigor. Interestingly, Styer et al. (1980) showed that sh 2 did not reduce seed vigor when seeds were harvested early in seed development (22 d postpollination) and then dried slowly under controlled conditions.

We conclude that the major differences seen in seedling vigor of sh 2 maize are due to the genotype of the endosperm. At the physiological level, it would appear that the association of the embryo with the mutant endosperm conditions a reduction in subsequent seed germination and vigor. The point at which this alteration in the embryo occurs is presently unknown, but it conceivably could occur at any stage during seed development or maturation. With this in mind, it is possible to improve emergence and apparent seed quality through mass selection of $S h 2$ genotypes without adversely affecting sweetness (Juvik et al., 1993). Potential pericarp uniformity and carbohydrate makeup might improve seed quality through reduced membrane porosity and leakage of materials from the seed.

\section{Literature Cited}

Barla-Szabo, G. and B. Dolinka. 1988. Complex stressing vigor test: A new method for wheat and maize seeds. Seed Sci. Technol. 16:63-73. Berger, R.D. and E.A. Wolf. 1974. Control of seedborne and soilborne mycoses of 'Florida Sweet' corn by seed treatment. Plant Dis. Rpt. 58:922-923.

Bernal-Lugo, I. and A.C. Leopold. 1992. Changes in soluble carbohydrates during seed storage. Plant Physiol. 98:1207-1210.

Bewley, J.D. and M. Black. 1978. Control processes in mobilization of stored reserves, p. 345. In: Physiology and Biochemistry of seeds. Springer Verlag, Berlin.

Bhave, M.R., S. Lawrence, C. Barton, and L.C. Hannah. 1990. Identification and molecular characterization of shrunken-2 CDNA clones of maize. Plant Cell 2:581-588.

Burr, B., F.A. Burr, and E.C. Matc. 1991. Data base for loci mapped in TxCM COxTx RI families. Maize Genet. Coop. Nwslt. 65:105-110.

Chen, Y. and J.S. Burris. 1990. The role of carbohydrates in desiccation tolerance and membrane behavior in maturing maize seeds. Crop Sci. 30:971-975.

Chern, G.S. and F.J. Sung. 1991. Prevention of injury during imbibition in shrunken-2 corn seeds by osmotic control of water uptake. Seed Sci. Technol. 19:469-476.

Crane, P.L., S.R. Miles, and J.E. Newman. 1959. Factors associated with varietal differences in rate of field drying in corn. Agron. J. 51:318-320.

Creech, R.G. 1956. Genetic control of carbohydrates synthesis in maize endosperm. Genetics 52:1175-1186.

Crowe, L.M., R. Mourdian, J.H. Crowe, S.A. Jackson, and C. Womersly. 1984. Effects of carbohydrates in membrane stability at lower water activities. Biochem. Biophys. Acta 769:141-150.

Dickinson, D.B. and J.G. Preiss. 1969. Presence of ADP-Glucose pyrophosphorylase in $s h 2$ and $b t 2$ mutants of maize endosperm. Plant Physiol. 44:1058-1062.

Fernandez, D.E. and L.A. Staehelin. 1985. Structural organization of ultrarapidly frozen barley aleurone cells actively involved in protein secretion. Planta 165:455-468.

Giroux, M., B. Smith-White, V. Gilmore, L.C. Hannah, and J. Preiss. 1995. The large subunit of the embryo isoform of ADP glucose pyrophosphorylase from maize. Plant Physiol. 108:1333-35.

Giroux, M. J., C. Boyer, G. Feix, and L.C. Hannah. 1994. Coordinated transcriptional regulation of storage product genes in the maize endosperm. Plant Physiol. 106:713-722.

Giroux, M. and L.C. Hannah. 1994. ADP-glucose pyrophosphorylase in shrunken 2 and brittle 2 mutants of maize. Mol. Gen. Genet. 243:400408.

Hannah, L.C. and O.E. Nelson. 1976 Characterization of ADPpyrophosphorylase from shrunken-2 and brittle-2 mutants of maize. Biochem. Genet. 14:547-560.

Harris, M.J. and D.A. DeMason. 1989. Comparative kernel structure of three endosperm mutants of Zea mays L. relating to seed viability and seedling vigor. Bot. Gaz. 150:50-62.

Juvik, J.A., M.C. Jargulo, J.M. Headrick, J.K. Pataky, and W.F. Tracy. 1993. Kernel changes in a Shrunken-2 maize population associated with selection for increased field emergence. J. Amer. Soc. Hort. Sci. 118:135-140.

Koster, K.L. 1991. Glass formation and desiccation tolerance in seeds. Plant Physiol. 96:302-304.

Koster, K.L. and A.C. Leopold. 1988. Sugar and desiccation tolerance in seeds. Plant Physiol. 88:829-832.

Martin, S., P.J. Loesch, and W.J. Wiser. 1980. A simplified technique for measuring pericarp thickness in maize. Maidica 25:9-16.

Murphy, J.B. and T.L. Noland. 1982. Temperature effects on seed imbibition and leakage mediated by viscosity and membranes. Plant Physiol. 69:428-431.

Murashige, T. and F. Skoog. 1962. A revised medium for rapid growth and bioassay with tobacco tissue culture. Plant Physiol. 15:473-497.

Parera, C.A. and D.J. Cantliffe. 1991. Improved germination and modified imbibition of shrunken-2 sweet corn by seed disinfection and solid matrix priming. J. Amer. Soc. Hort. Sci. 116:942-945.

Parera, C.A., D.J. Cantliffe, P.J. Stoffella, and B.T. Scully. 1995. Field emergence of shrunken-2 corn predicted by single- and multiple-vigor laboratory tests. J. Amer. Soc. Hort. Sci. 120:128-132.

Parrish, D.J. and A.C. Leopold. 1978. On the mechanism of aging in soybean seeds. Plant Physiol. 61:365-368.

Preiss, J. 1978. Regulation of ADP glucose pyrophosphorylase, p. 432. In: A. Meister (ed.). Advances in enzymology and related areas of molecular biology. Wiley, New York.

Roman, H. and A.J. Ullstrup. 1951. The use of A-B translocation to locate genes in maize. Agron. J. 43:450-453.

Sanwo, M.M. and D.A. DeMason. 1992. Characteristics of -amylase 
during germination of two high-sugar sweet cultivars of Zea mays L. Plant Physiol. 99:1184-1192.

Schoettle, A.W. and A.C. Leopold. 1984. Solute leakage from artificially aged soybean seed after imbibition. Crop Sci. 24:835-838.

Styer, R.C. and D.J. Cantliffe. 1983. Relationship between environment during seed development and seed vigor of two endosperm mutants of corn. J. Amer. Soc. Hort. Sci. 108:717-720.

Styer, R.C. and D.J. Cantliffe. 1984a. Dependence of seed vigor during germination on carbohydrate source in endosperm mutant of maize. Plant Physiol. 76:196-200.

Styer, R.C. and D.J. Cantliffe. 1984b. Infection of two endosperm mutants of sweet corn by Fusarium moniliforme and its effect on seedling vigor. Phytopathology 74:189-194.
Styer, R.C., D.J. Cantliffe, and L.C. Hannah. 1980. Differential seed and seedling vigor in shrunken-2 compared to three other genotypes of corn at various stages of development. J. Amer. Soc. Hort. Sci. 105:329-332.

Tracy, W.F. and J.A. Juvik. 1988. Electrolyte leakage and seed quality in a shrunken-2 maize selected for improved field emergence. HortScience 23:391-392.

Tsai, C.Y. and O.E. Nelson. 1966. Starch deficient maize mutant lacking adenosine diphosphate glucose pyrophosphorylase activity. Science 151:341-343.

Wann, E.V. 1980. Seed vigor and respiration of maize kernels with different endosperm genotypes. J. Amer. Soc. Hort. Sci. 105:31-34.

Woodstock, L.W. 1988. Seed imbibition: a critical period for successful germination. J. Seed Technol. 12:1-15. 